CIRCUIT BOARD ROUTING ATTACHMENT

FOR FERMILAB GERBER PLOTTER

Carl Lindenmeyer

May 10, 1984 
A new and potentially important method of producing large circuit boards has been developed at Fermilab. A Gerber Flat Bed Plotter with an active area of $5^{\prime} \times 16^{\prime}$ has been fitted with a machining head to produce a circuit board without the use of photography or chemicals.

The modifications of the Gerber Plotter do not impair its use as a photoplotter or pen plotter, the machining head is merely exchanged with the standard attachments. The modifications to the program are minimal, this will be described in another report.

The machining head is fitted with an air bearing motorized spindle driven at a speed of $40,000 \mathrm{r} . \mathrm{p} . \mathrm{m}$. to $90,000 \mathrm{r.p.m}$. The spindle also is provided with air bearings on its outside diameter, offering frictionless vertical travel guidance. Vertical travel of the spindle is driven by a spring return single acting air cylinder. An adjustable hydraulic damper slows the spindle travel near the end of its downward stroke. Two programmable stops control spindle down stroke position, and limit switches are provided for position feedback to the control system. A vacuum system collects chips at the cutter head. No lubrication or regular maintenance is required.

The circuit board to be fabricated is supported on a porous plastic mat which allows table vacuum to hold the board in place while allowing the cutters or drills to cut through the board without damaging the rubber platen of the plotter. The perimeter of the board must be covered to the limits of the table vacuum area used to prevent excessive leakage.

This device is intended to perform 4 different operations in the production of a circuit board:

\section{1) Routing}

Circuit paths and/or charge collection areas are isolated from other copper clad areas of the board by cutting through the copper with a small cutter rotating at a speed of 90,000 r.p.m. For one ounce copper ( $0.0014^{\prime \prime}$ thick) a cut $0.002^{\prime \prime}$ to 0.004 " deep is adequate. The width of the cut can be 0.010" to 0.050" wide depending on the cutter used. Initial test cuts utilized a 4 flute cutter with a width of $0.040^{\prime \prime}$ and a cut depth of $0.004 "$ cutting speed was approximately 11" per second. The depth of cut is controlled by presetting the cutter length in a special presetting fixture. The spindle rides on the copper surface supported by a hardened shoe, allowing the spindle to follow height variations of the work surface.

\section{2) Drilling}

Drills with a 1/8" diameter shank must be used. Minimum drilling speed is 40,000 r.p.m. to ensure actuation of the centrifugal collet, maximum speed can be 90,000 r.p.m. and is presently set at 90,000 r.p.m. Maximum drill size should be limited to about 1/8" diameter, minimum size is only limited by the available 
speed and is expected to be no problem. Cycle speed for drilling is affected by many variables, initial tests with a 1/8" diameter drill produced holes at a rate of 1.6 holes per second.

\section{3) "Slow" Routing}

Similar to circuit routing except it is intended for cutting a larger path at a lower r.p.m., used to clear copper from larger areas of the circuit board. Minimum speed is 40,000 r.p.m. permitting a larger cutter size. As with circuit routing, the depth of cut is controlled with a preset cutter length and by supporting the spindle on the copper surface. Initial test were with a 1/8" diameter 4 flute cutter at 90,000 r:p.m., the feed speed was $11 "$ per second.

\section{4) Contouring}

The circuit board outline can be profiled using a cutter set to a depth slightly below the board underside. During this operation the support shoe on the spindle is approximately $1 / 32$ " above the copper surface. Using a 1/8" diameter 4 flute cutter at 90,000 r.p.m., feed speed was $11 / 2^{\prime \prime}$ per second at initial tests.

The first use for this device will be fabricating charge collection panels for the E-706 hadron calorimeter from 1/32" thick $4^{\prime} \times 10^{\prime}$ G-10, other similar applications are bound to surface as more experimenters became aware of the capabilities of this machine. The speeds and feeds as well as cutter geometry can be optimized as more experience is gained in operation. This device is primarily unique because of its size and speed. A number of small machines operating in a similar way are offered by K.B.C. Automated Process Systems Limited of Toronto, Canada. Maximum board size is 10.24" x 14.18", routing speed is $0.4 "$ per second and approximate 2.5 seconds per hole including travel.

Programming data is being prepared and several people are trained in operation of this machine. The machine is located in Lab 8 and is under the control of Research Services Division. Evaluation of proposed uses can be obtained from Ed Arko, Carl Lindenmeyer of the Physics Department, or Mike Glaubman of E-706. 